\title{
Predictive Value of miR-146a rs2431697 Polymorphism to Myelofibrosis Progression in Patients with Myeloproliferative Neoplasm
}

\author{
Salah Aref ${ }^{1 *}$, Doaa Atia ${ }^{1}$, Ahmed Al Tantawy ${ }^{2}$, Mohamed Al Boghdady ${ }^{3}$, Enas \\ Gouda ${ }^{1}$
}

\begin{abstract}
Background: Bone marrow myelofibrosis (BMF) that develop on top of Polycythaemia vera (PV) and essential thrombocythemia leads to shortening of the patient's overall survival. This study aimed to address the impact of miR146a rs2431697 polymorphism on inflammatory biomarkers and genes expression and the hazards of myelofibrosis progression. Patients and Methods: The study included 88 myeloproliferative neoplasm (40 PV; 27 ET; 21 MF) and 90 healthy controls. For all investigated subjects miR-146a rs 2431697 genotypes were identified by sequencing and the expression of miR-146a; IL-1 $\beta$; NF-kB; a NOD-like receptor family, pyrin domain containing 3 (NLRP3) genes were estimated by real time PCR. Results: miR146a genotypes revealed that there was significant association between TT and TC genotypes with MF. The degree of miR146a expression was significantly reduced in MF as compared to both PV and ET. In contrast; the levels of IL-1 $\beta$; NF- $\mathrm{KB}$; NLRP3 genes expression were significantly elevated in MF patients group as compared to PV and ET patients' group. Multivariate analysis identified TT genotype as poor predictor of MF progression. Conclusion: miR-146a rs2431697 TT genotype is associated with high risk of MF progression in MPN patients. Targeting of IL-1 $\beta$; NF- $\kappa B$; NLRP3 genes might help in hindering of MF progression in MPN patients,
\end{abstract}

Keywords: miR-146a- IL-1 $\beta$ - NF-kB- NLRP3- MPN- Myelofibrosis

Asian Pac J Cancer Prev, 22 (11), 3585-3589

\section{Introduction}

The Philadelphia negative Myeloproliferative neoplasms (MPNs) (BCR-ABL-ve MPNs) are the most frequent diseases among the myeloproliferative disorders. MPNs are subcategorized into 3 subtypes that include polycythemia Vera (PV), essential thrombocytosis (ET), and Myelofibrosis (MF). MPN is characterized by progressive production of mature functioning terminal cells (Red cells and Platelets). During the progressive course of PV and ET; some of the patients transformed into MF which usually associated with complications namely thrombosis, hemorrhages, and worse complication which is transformation to acute myeloid leukemia (AML) (Vainchenker and Kralovics, 2017).

The discovery of driver mutations namely JAK2, CALR, and MPL reveal that activated JAK-STAT signaling is main trigger of $\mathrm{MF}$, supporting a rationale for JAK inhibition. However, JAK inhibition alone is insufficient for long-term remission and offers modest, if any, disease-modifying effects (Zhou et al., 2020).

It is become clear that there is a close link between chronic inflammation and MPN pathogenesis. The expression of IL-1 which is an inflammatory cytokine was found to be increased in MPN. Also, the incidence of MPN patients was higher among patients suffering from autoimmune or inflammatory (Lussana and Rambaldi, 2017; Wang and Zuo, 2019). Moreover; it has been reported that the main source of inflammatory cytokines are neoplastic clone and its differentiated progeny. These cytokines are released in the tumor microenvironment and leads to cytokine storm within the bone marrow niche which subsequently induces to marrow fibrosis (Lussana and Rambaldi, 2017). The time from start of disease to transformation to MF is usually differed being shorter in PV than ET and according to JAK2 allele burden and CALR mutations as well as cooperation of myeloid genes (Ferrer-Marín etal., 2020).

miR-146a-5p has been described as a negative regulator in innate immune and inflammatory responses mediated by Toll like receptor 4 . Signaling downstream of TLR4 predominantly activates NF- $\kappa \mathrm{B}$ after sequential activation of intermediate targets namely IRAK1 and TRAF6 (Taganov et al., 2006), which drives immune responses

${ }^{1}$ Hematology Unit, Department of Clinical Pathology, Faculty of Medicine, Mansoura University, Egypt. ${ }^{2}$ Medical Oncology Unit, Mansoura University Oncology Center (MUOC) Mansoura University; Egypt. ${ }^{3}$ Hematology Unit, Mansoura University Oncology Center (MUOC), Mansoura University,Egypt.*For Correspondence: salaharef@mans.edu.eg 
like leukocyte recruitment and pro-inflammatory cytokine production, exacerbating any disease (Jin et al., 2011). Also; it is the unique miRNA related to immune and inflammatory response with polymorphisms (miRSNPs) that influence its expression levels: rs2431697 ( 1 kb upstream of of miR146A gene) and rs2910164 (within miR146A gene) (Lofgren et al., 2012). Interestingly, these two miRSNPs have been related with inflammatory response associated with certain diseases (Ramkaran et al., 2014).

$\mathrm{NF}-\kappa \mathrm{B}$ has been proposed as a key mediator of inflammation-induced carcinogenesis (Grivennikov et al 2010, Karin and Greten, 2005). As in other malignancies, $\mathrm{NF}-\kappa \mathrm{B}$ activation is detected in MF (Chorzalska et al., 2012). miR-146a is a significant brake on autoimmunity, myeloproliferation, and cancer in mice ( Boldineta., 2011).

This study aimed to delineate the association between miR-146a (rs2431697) polymorphisms and certain inflammatory biomarkers and the hazards of myelofibrosis progression.

\section{Materials and Methods}

\section{Patients and Methods}

All patients diagnosed as MPN who presented at Mansoura University Oncology Center (MUOC) between January 2015 up to September 2020 were included in cohort prospective study. The study included 88 patients (median age 62 years; age range 45-76) (45 Male; 43 Female). MPN (40 PV; 27ET; 21MF). The diagnosis of MPN was carried out according to the 2016 World Health Organization (WHO) (Grinfeld et al 2018). The MPN diagnostic subtypes comprised MPN (40 PV; 27ET; $21 \mathrm{MF}$ ) recruitment at Mansoura University Oncology Center. Ninety healthy controls matched for age and sex with negative past history of hematological disorders were included as controls. EDTA-anticoagulated bone marrow samples from MPN patients and peripheral blood from healthy donors were collected. The investigated persons characteristics were shown in Table1. The study was approved by Mansoura University IRB committee.

\section{DNA extraction and genotyping}

The SNP rs2431697 is situated in the intergenic region between the Pituitary tumor-transforming gene 1 (PTTG1) and miR146a genes. The Genomic DNA was extracted from the peripheral blood samples collected in EDTA test tubes using a DNA Purification Kit (Promega, Madison, WI, USA). miRNA-146a rs2910164 C > G polymorphism genotyping was analyzed. In brief, a $150 \mathrm{ng}$ DNA sample was heated to $98^{\circ} \mathrm{C}$ and held for 5 minutes. The ligation reaction was carried out in an ABI 2720 thermal cycler. Then, a 48-plex fluorescence polymerase chain reaction (PCR) was conducted. In an ABI 3730XL sequencer, capillary electrophoresis was harnessed to analyze the PCR products. Gene Mapper 4.1 software (Applied Biosystems, Foster City, CA, USA) was used to read the information of the genotype. For quality control, different technicians genotyped $4 \%$ of the genomic DNA samples that were randomly selected. And, the results were in full accord with the findings of the first assays.

$R N A$ isolation, $c D N A$ synthesis and quantitative real-time $P C R$

Fresh peripheral blood samples were collected in tubes containing EDTA. Lysis of red cells were done immediately and about $1 \mu \mathrm{g}$ total RNA from each blood samples was extracted using Trizol (Invitrogen) and after that complementary DNA (cDNA) was synthesized from total RNA using Superscript II reverse transcriptase (Invitrogen) and oligo (dT) primers according to manufacturer instructions.

PCR was performed with the following thermo-cycling conditions: An initial $5 \mathrm{~min}$ at $95^{\circ} \mathrm{C}$, followed by 40 cycles of $95^{\circ} \mathrm{C}$ for $30 \mathrm{sec}, 55^{\circ} \mathrm{C}$ for $30 \mathrm{sec}$ and $72^{\circ} \mathrm{C}$ for $30 \mathrm{sec}$.

The real-time PCR system contained $5 \mu$ of $2 \times$ SYBR Green Real-time PCR Master Mix, $0.8 \mu$ l of the forward and reverse primers and $1 \mu \mathrm{l}$ of cDNA, in a final volume of $10 \mu \mathrm{l}$. The relative mRNA expression of NLRP3, NF$\kappa \mathrm{B} 1$, IL-1 $\beta$, was determined by ABI Prism 7500 realtime PCR system (Applied Biosystems, Foster City, CA, USA). Primers sequences used for reverse transcriptionquantitative polymerase chain reaction analysis (15). NFкB1 Forward (F) 5'-TCCAGACCAACAACAACCCC-3', Reverse (R) GATCTTCTCGGCAGTGT-3`; for NLRP3 F. 5'-CAGACTTCTGTGTGTGGGACTGA-3', R. 5'-TCCTGACAACATGCTGATGTGA-3 '; For GAPDH F, 5'-GCTCTCTGCTCCTCCTGTTC; R. GTTGACTCCGACCTTCACCT-3 '; for miR146a F. 5'-TGAGAACTGAATTCCATGGGTT-3', R. 5'-GCTGTCAACGATACGCTACGTAACG; for U6 F. 5' - GCTTCGGCAGCACATATACTAAAAT-3', R. 5'-CGCTTCACGAATTTGCGTGTCAT-3'.

All experiments were done in duplicate. The number of GAPDH transcript was used as an internal control for IL-1 $\beta$; NF- $\kappa$ B1; NLRP3 and U6 was used as internal control for miR-146a. Transcript levels were expressed as arbitrary units and were calculated using the comparative threshold cycle method. Melting curve analysis was applied in all the PCR products.

\section{Statistical analyses}

Continuous variables are presented as the mean $\pm \mathrm{SD}$ and categorical variables are presented as percentages. Comparisons of categorical variables between groups were carried out using the $\chi^{2}$ test for tables, while numerical variables were compared using the two-tailed Student's t test or Mann-Whitney U test, where appropriate. Shapiro-Wilk test and Levene's test were used to check normal distribution and homoscedasticity assumptions, respectively. The impact of TT on Transformation to MF is assessed by was evaluated by Cox regression.

\section{Results}

Gene frequency of miR-146a rs2431697 polymorphism in different studied groups

The frequency of miR-146a rs2431697 genotypes in $\mathrm{PV}$ and ET did not significantly different $(\mathrm{P}>0.05)$. The $\mathrm{T}$ allele of rs2431697 is more frequent in MF case group as compared with the control group. The genotype TT is 
DOI:10.31557/APJCP.2021.22.11.3585 miR-146a Polymorphism and MF Progression in MPN

Table 1. Main Patients Data of PV and ET Patients at the Time of Diagnosis

\begin{tabular}{|c|c|c|c|c|c|}
\hline Parameters & $\begin{array}{l}\text { Control } \\
(\mathrm{n}=90)\end{array}$ & $\begin{array}{c}\mathrm{PV} \\
(\mathrm{n}=40)\end{array}$ & $\begin{array}{c}\mathrm{ET} \\
(\mathrm{n}=27)\end{array}$ & $\begin{array}{c}\text { MF (Post PV and ET) } \\
(\mathrm{n}=21)\end{array}$ & $\begin{array}{c}\mathrm{P} \\
\text { Value }\end{array}$ \\
\hline Age/years Median (Range) & $61(45-76)$ & $56(45-70)$ & $53(45-65)$ & $62(52-72)$ & $\leq 0.001$ \\
\hline \multicolumn{6}{|l|}{ Sex } \\
\hline Male, no (\%) & $46(51.1)$ & $19(47.5)$ & $14(46.4)$ & $12(5.5)$ & 0.9 \\
\hline Female, no (\%) & $44(48.9)$ & $21(52.5)$ & $13(53.6)$ & $9(45.5)$ & \\
\hline Hemoglobin, g/l & $13.5 \pm 1.2$ & $17.5 \pm 1.7$ & $14.1 \pm 1.5$ & $16.2 \pm 4.5$ & $\leq 0.001$ \\
\hline WBC count, $\times 10^{9} / 1$ & $8.2 \pm 2.2$ & $16.5 \pm 4.4$ & $8.7 \pm 3.2$ & $9.23 \pm 1.8$ & $\leq 0.001$ \\
\hline Platelet count, $\times 10^{9} / 1$ & $230 \pm 32$ & $597 \pm 288$ & $816 \pm 289$ & $420 \pm 42$ & $\leq 0.001$ \\
\hline JAK2 mutated, n (\%) & - & $40(100 \%)$ & $14(52 \%)$ & $16(76 \%)$ & $\leq 0.001$ \\
\hline CALR mutated, n (\%) & - & $22(55 \%)$ & $23(51.8 \%)$ & & \\
\hline MPL mutated, n (\%) & - & $1(2.5 \%)$ & $2(7.4 \%)$ & & \\
\hline Follow up time (years) & & 6 & 6 & & \\
\hline
\end{tabular}

Table 2. miR-146a rs2431697 Polymorphisms in Different Studied Group

\begin{tabular}{lcccc}
\hline Genotypes & Control $(\mathrm{n}=90)$ & PV $(\mathrm{n}=40)$ & ET $(\mathrm{n}=27)$ & MF $(\mathrm{n}=21)$ \\
\hline CC & $20(22.2)$ & $10(25.0)$ & $7(25.9)$ & $5(23.8)$ \\
TC & $59(65.6)$ & $20(50.0)$ & $12(44.4)$ & $5(23.8)$ \\
TT & $11(12.2)$ & $10(25,0)$ & $8(29.6)$ & $11(52.4)$ \\
P value & P1 $>0.05$ & P2 $>0.05$ & P3 $>0.05$ & P4 $\leq 0.001$ \\
\hline
\end{tabular}

P1, genotypesin Controlvs PV; P2, genotypes in controlvs ET; P3, genotypes in PV vs ET; P4, genotypes in MF vs PV and ET

more frequent in MF group as compared to both PV; ET and control group $(\mathrm{P}=0.001)$ (Table 2$)$.
Expression of genes involved in NLRP3 inflammasome signaling were increased in $M P N$

The expression of IL-1 $\beta$; NF-kB1 and NLRP3 which

Table 3. Genes Expressions of Inflammatory Mediators in All Studied Persons

\begin{tabular}{lccccc}
\hline & $\begin{array}{c}\text { Control }(\mathrm{n}=90) \\
\text { Median (min-max) }\end{array}$ & $\begin{array}{c}\text { PV (n=40) } \\
\text { Median (min-max) }\end{array}$ & $\begin{array}{c}\text { ET (n=27) } \\
\text { Median (min-max) }\end{array}$ & $\begin{array}{c}\text { MF (n=21) } \\
\text { Median (min-max) }\end{array}$ & P value \\
\hline IL-1 3 & $0.35(0.1-0.95)$ & $1.3(0.2-2.4)$ & $1.2(0.2-2.3)$ & $1.7(0.2-2.6)$ & 0.002 \\
NF-kB1 & $0.4(0.2-1.2)$ & $0.7(0.2-2.1)$ & $0.7(0.2-1.4)$ & $0.9(0.2-1.9)$ & $\leq 0.001$ \\
NLRP3 & $0.44(0.1-1.33)$ & $1.2(0.2-3.4)$ & $0.6(0.5-2.4)$ & $0.9(0.2-1.9)$ & $\leq 0.001$ \\
miR-146a & $1.2(0.77-2.23)$ & $0.55(0.45-2.44)$ & $0.48(0.34-1.5)$ & $0.22(0.0-0.92)$ & 0.001 \\
\hline
\end{tabular}

Table 4. Impact of miR-146a Genotypes on Inflammatory Genes Expression in All Studied Groups

\begin{tabular}{|c|c|c|c|c|}
\hline & $\begin{array}{c}\text { Control } \\
\text { Median (min-max) }\end{array}$ & $\begin{array}{c}\text { PV } \\
\text { Median (min-max) }\end{array}$ & $\begin{array}{c}\text { ET } \\
\text { Median (min-max) }\end{array}$ & $\begin{array}{c}\text { MF } \\
\text { Median (min-max) }\end{array}$ \\
\hline \multicolumn{5}{|l|}{ IL-1 $\beta$} \\
\hline $\mathrm{CC}$ & $0.18(0.1-0.25)$ & $0.47(0.24-1.1)$ & $0.56(0.2-0.95)$ & $0.4(0.2-0.6)$ \\
\hline $\mathrm{TC}$ & $0.6(0.22-0.87)$ & $1.4(0.45-1.95)$ & $1.1(0.2-1.4)$ & $1.6(0.6-1.7)$ \\
\hline TT & $0.77(0.54-0.95)$ & $2.1(1.4-2.4)$ & $2.0(1.3-2.3)$ & $2.1(1.6-2.6)$ \\
\hline$P$ value & $\leq 0.001$ & $\leq 0.001$ & $\leq 0.001$ & $\leq 0.001$ \\
\hline \multicolumn{5}{|l|}{ NF-кB1 } \\
\hline $\mathrm{CC}$ & $0.39(0.21-0.95)$ & $0.32(0.22-0.7)$ & $0.34(0.2-0.7)$ & $0.19(0.15-0.44)$ \\
\hline $\mathrm{TC}$ & $0.65(0.23-0.92)$ & $0.8(0.2-2.1)$ & $0.65(0.2-0.9)$ & $0.67(0.2-0.9)$ \\
\hline TT & $0.94(0.43-1.2)$ & $1.2(0.8-1.9)$ & $1.2(1.0-1.4)$ & $1.4(0.9-1.9)$ \\
\hline P value & $\leq 0.001$ & $\leq 0.001$ & $\leq 0.001$ & $\leq 0.001$ \\
\hline \multicolumn{5}{|l|}{ NLRP3 } \\
\hline $\mathrm{CC}$ & $0.13(0.1-0.23)$ & $0.58(0.23-0.66)$ & $0.7(0.5-1.1)$ & $0.7(0.5-1.0)$ \\
\hline $\mathrm{TC}$ & $0.55(0.12-0.88)$ & $1.4(0.7-2.2)$ & $1.3(1.0-1.8)$ & $1.8(0.9-1.9)$ \\
\hline TT & $0.8(0.22-1.33)$ & $2.8(2.0-3.4)$ & $2.1(1.9-2.4)$ & $2.9(2.4-3.2)$ \\
\hline$P$ value & $\leq 0.001$ & $\leq 0.001$ & $\leq 0.001$ & $\leq 0.001$ \\
\hline
\end{tabular}


Table 5. Correlation between miR146a and Inflammatory Genes Expression in Total MPN Group of Patients

\begin{tabular}{cccc}
\hline & IL-1 & NF- $\kappa B 1$ & NLRP3 \\
miRNA146a & $\mathrm{r}=-0.632 \mathrm{P}<0.01$ & $\mathrm{r}=-0.574 \mathrm{P}<0.01$ & $\mathrm{r}=-0.483 \mathrm{P}<0.01$ \\
\hline
\end{tabular}

Table 6. Association between miR-146a rs2431697 Polymorphism Genotype and PV and ET Transformation to MF

\begin{tabular}{lccccc}
\hline Genotypes & PV $(\mathrm{n}=40)$ & $\mathrm{ET}(\mathrm{n}=27)$ & $\mathrm{MF}(\mathrm{n}=21)$ & $\mathrm{MF}$ vs.PV & MF vs.ET \\
& $\mathrm{N}(\%)$ & $\mathrm{N}(\%)$ & $\mathrm{N}(\%)$ & $\mathrm{OR}(95 \% \mathrm{CI})$ & OR (95\% CI) \\
\hline CC & $10(25.0)$ & $7(25.9)$ & $5(23.8)$ & $\mathrm{r}(1)$ & $\mathrm{r}(1)$ \\
$\mathrm{TC}$ & $25(62.5)$ & $12(44.5)$ & $5(23.8)$ & $0.3(0.1-1.4)$ & $0.5(0.1-2.1)$ \\
$\mathrm{TT}$ & $5(12.5)$ & $8(29.6)$ & $11(52.4)$ & $3.5(0.8-16.7)$ & $1.6(0.4-7.0)$ \\
\hline
\end{tabular}

OR, Odds ration; $r$, reference category; $\mathrm{CI}$,Confidence interval

are intracellular mediators are elevated in MPN subgroups as compared to controls (IL- $1 \beta ; \mathrm{P}=0.002 ; \mathrm{NF}-\kappa \mathrm{B} 1$ $\mathrm{P}=\leq 0.001 ; \mathrm{NF}-\kappa \mathrm{B} 1 \mathrm{P}=\leq 0.001$; NLRP3 $\mathrm{P}=0.001)$. The highest levels were detected in MF group (Table 3).

Association of the miR-146a rs2431697 SNP with miR-146a; IL-1 $\beta ; N F-\kappa B 1$ and NLRP3 genes expression

It is evident that TT genotype is associated with lowest levels of IL-1 $\beta$; NF- $\mathrm{kB} 1$ and NLRP3 genes expression in all studied MPN subgroups as compared to TC and CC genotypes $(\mathrm{P}<0.001$ for all) (Table 4$)$.

Correlation studies between T allele of rs2431697 and inflammatory genes expression in MPN subgroups

Correlation studies revealed that there is significant positive correlation between the 3 parameters in $\mathrm{PV}$

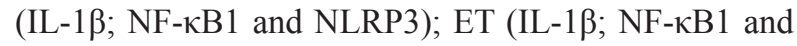

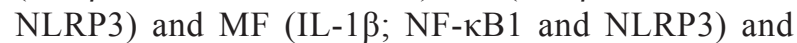
significant negative correlation between $\mathrm{T}$ allele and miR-146a (Table 4).

Correlation between mRNA146a expression and inflammatory mediators in total MPN group

The statistical analysis in Table 5 revealed that there is negative significant correlation between mRNA146a expression and IL-1 $\beta$; NF- $\mathrm{kB} 1$ and NLRP3.

Odds ratio of miR-146a rs2431697 polymorphism genotype on $P V$ and ET transformation to $M F$

The TT genotype of miR-146a rs2431697 polymorphism was associated with the risk of transformation of $\mathrm{PV}$ and $\mathrm{ET}$ to $\mathrm{MF}$ with a OR: $3.5(\mathrm{CI}$ : 0.8-16.7) in PV MPN subgroup and OR: 1.6(CI: 0.4-7.0) in ET and MPN subgroup respectively (Table 6).

\section{Discussion}

The cardinal role of inflammatory process in the pathogenesis of MPN had emerged in many studies. Ferrer-Marín et al. (2020) and Masselli et al.(2019) have recently identified the $2518 \mathrm{~A} / \mathrm{G}$ polymorphism in MCP-1, the main chemotactic factor for monocyte migration to sites of inflammation, as a potential genetic predisposition factor for secondary MF (Masselli et al., 2019).

The relationship between miR-146a and inflammatory process that is associated with MPN has been demonstrated in many previous reports. This reports stated that miR-146a modify the inflammatory process through accentuating NF-kB signaling (Taganov et al., 2006 and Guglielmelli etal., 2007). Likewise; symptoms of chronic inflammation that was described in MPN patients was attributed to increased inflammatory mediators in the serum of those patients. However; the relation between these inflammatory markers and progression to MF is not fully elucidated.

Our results showed that the rs2431697 TT genotype is frequently detected in MF subtypes of MPN patients as compared to controls. Similar finding was reported by Zhou et al., (2020). Lofgren et al., (2012) demonstrated that SNPs rs2431697 modify miR-146a expression levels.

The expression of inflammatory mediators including

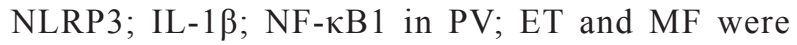
significantly elevated as compared to control group. Meanwhile the expression of these markers was significantly higher in MF group as compared to both PV and ET groups. These findings are similar to that reported by Zhou et al (2020) who found that inflammasome-related genes (NLRP3, NF- $\kappa \mathrm{B} 1, \mathrm{IL}-1 \beta$ ) were highly expressed in BM cells from MPN patients and the increased expression was associated with JAK2V617F mutation, white blood cell counts and splenomegaly and suggested that these findings point out for novel biomarkers which may be a suggested target for MPN control.

The association between rs2431697 TT genotype and inflammatory mediators' expression has been evaluated in the current study. Our results revealed that the TT genotypes are associated with high expression of inflammatory-related genes namely NLRP3, NF-KB1, and IL-1 $\beta$. These findings are in parallel with that reported in a previous recent study (Ferrer-Marín etal., 2020).

Importantly, our findings suggest that the rs 2431697 TT genotype not only increases susceptibility to secondary MF, but may also be considered a marker for early progression. These findings strongly suggest that the rs2431697 genotype represents a new prognostic factor for MPN, and may point to the development of new therapeutic strategies based on the use of novel agents such as miRNA mimics.

The limitation of this study is small sample size of our cohort of MPN patients. Large scale study is recommended to validate our findings.

In conclusion our findings demonstrated the impact 
of miR-146a polymorphism on its expression and subsequently the progression of MPN to MF. This is through down regulation of miR-146a expression and up regulation of the genes (NLRP3, NF- $\kappa B 1, I L-1 \beta$ ) that control inflammations. Targeting NLRP3, NF- $\kappa$ B1, IL-1 $\beta$ genes might hinder MF progression in MPN patients.

\section{Author Contribution Statement}

Salah Aref: Conception and study design and Manuscript revision; Doaa Atia: Laboratory work; Interpretation and analysis of data; Ahmed Al Tantawy: Preparation of the manuscript and Revision for Important intellectual; Mohamed Al Boghdady: Clinical assessment of Patients; Enas Gouda: Laboratory work; Interpretation and analysis of data.

\section{Acknowledgments}

The present study is the result of free research project and approved by ethical committee of Mansoura Faculty of Medicine. The study was funded by the authors them self. The authors would like to thank the technicians in the Molecular Hematology laboratory at Mansoura University Oncology Center (MUOC) laboratories. The data of the present study is available upon request. The authors declare that there is no any conflict of interest.

\section{References}

Boldin MP, Taganov KD, Rao DS et al (2011). miR-146a is a significant brake on autoimmunity, myeloproliferation, and cancer in mice. J Exp Med, 208, 1189-201.

Chorzalska AD, Morgan J, Treaba DO et al (2016). Bone marrow-specific loss of ABI1 induces myelofibrosis through a mechanism involving activation of NFkB. Blood, 22, 603 .

Ferrer-Marín F, Arroyo A, GEMFIN Group (2020). miR-146a rs2431697 identifies myeloproliferative neoplasm patients with higher secondary myelofibrosis progression risk. Leukemia, 34, 2648-59.

Guglielmelli P, Tozzi L, Pancrazzi A, et al (2007). MicroRNA expression profile in granulocytes from primary myelofibrosis patients. Exp Hematol, 35, 1708-18.

Hasselbalch HC (2012) Perspectives on chronic inflammation in essential thrombocythemia, polycythemia Vera, and myelofibrosis: is chronic inflammation a trigger and driver of clonal evolution and development of accelerated atherosclerosis and second cancer?. Blood, 119, 3219-25.

Grivennikov SI, Greten FR, Karin M (2010). Immunity, inflammation, and cancer. Cell, 140, 883-99.

Grinfeld J, Nangalia J, Baxter EJ, et al (2018). Classification and personalized prognosis in myeloproliferative neoplasms. $N$ Engl J Med, 379, 1416-30.

Jin J, Samuvel DJ, Zhang X, et al (2011). Coactivation of TLR 4 and TLR2/ 6 coordinates an additive augmentation on IL-6 gene transcription via p38MAPK pathway in U937 mononuclear cells. Mol Immunol, 49, 423-32.

Karin M, Greten FR. (2005). NF-kappa B: linking inflammation and immunity to cancer development and progression. Nat Rev Immunol, 5, 749-59.

Lofgren SE, Frostegard J, Truedsson L, et al. (2012). Genetic association of miRNA-146a with systemic lupus erythematosus in Europeans through decreased expression of the gene. Genes Immune, 13, 268-74.
Lussana F, Rambaldi A (2017). Inflammation and myeloproliferative neoplasms. J Autoimmun, 85, 58-63.

Masselli E, Carubbi C, Cambo B, et al (2018). The -2518 A/G polymorphism of the monocyte chemoattractant protein-1 as a candidate genetic predisposition factor for secondary myelofibrosis and biomarker of disease severity. Leukemia, 32, 2266-70.

Ramkaran P, Khan S, Phulukdaree A, et al (2014). miR-146a polymorphism influences levels of miR-146a, IRAK-1, and TRAF-6 in young patients with coronary artery disease. Cell Biochem Biophys, 68, 259-26.

Taganov KD, Boldin MP, Chang KJ et al (2006). Baltimore D. NF-kappaB-dependent induction of microRNA miR-146, an inhibitor targeted to signaling proteins of innate immune responses. Proc Natl Acad Sci U S A, 103, 12481-86.

Vainchenker W, Kralovics R (2017). Genetic basis and molecular pathophysiology of classical myeloproliferative neoplasms. Blood, 129, 667-79.

WangY, Zuo X (2019) Cytokines frequently implicated in myeloproliferative neoplasms. Cytokine, X1, 100005.

Zhou Y, ShuxinYan S, NaLiu Na He, et al (2020). Genetic polymorphisms and expression of NLRP3 inflammasomerelated genes are associated with Philadelphia chromosomenegative myeloproliferative neoplasms. Hum Immunol, 81, 606-13.

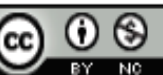

This work is licensed under a Creative Commons AttributionNon Commercial 4.0 International License. 